\title{
Maximizing The Impact Of Improvement Efforts On Customer Satisfaction
}

\author{
Dooyoung Shin, Minnesota State University, Mankato, Mankato, USA \\ Jon Kalinowski, Minnesota State University, Mankato, Mankato, USA \\ Sung Kim, Minnesota State University, Mankato, Mankato, USA \\ In-Jae Kim, Minnesota State University, Mankato, Mankato, USA
}

\begin{abstract}
$^{1}$
When a customer satisfaction survey consists of a large number of attributes (questionnaire items), determination of critical attributes that would make the biggest impact on customers' overall satisfaction could be important, but very tedious and time-consuming process. Even though the critical attributes are identified, the improvement efforts toward these attributes are often misdirected and wasted because of the mismatch between the improvement efforts and the critical needs of the affected customer group. This paper introduces a method with which improvement efforts can be tailored to the needs of the customer group who could bring the most impactful influence on improving customer satisfaction. For the critical attribute considered, the percentage of customers who assigned a specific satisfaction rating is obtained, and the cumulative percentages of customers are examined and the target group of customers to whom the improvement efforts would be tailored is identified. The piecewise linear approximation method is also discussed to the non-linear relationship of the attribute, which also may help determine the target customer group. The overall shape of the piecewise function and the slopes at the line segments may be used in determining which attributes are satisfaction-maintaining or satisfaction-enhancing, and where and how the improvement efforts should be focused in order to maximize the effectiveness of the improvement efforts.
\end{abstract}

Keywords: Satisfaction-Enhancing Attributes; Satisfaction-Maintaining Attributes; Piecewise Linear Approximation

\section{INTRODUCTION}

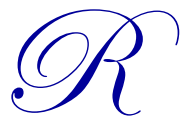

ecognizing that satisfied customers are the key to the success, many companies make strenuous efforts to achieve high levels of customer satisfaction. Companies continually monitor and examine the experiences, opinions, critical needs and suggestions of their customers in order to improve customer satisfaction. Customer satisfaction on a product or service is often shaped based on satisfaction with various attributes that comprise the significance of the product or service. In order to improve customers' overall satisfaction that may be closely related to customer retention and profitability, many companies make concerted to improve performance on various product or service attributes (Hanson (1992), Wittink and Bayer (1994), Mittal, Ross, and Baldasare (1998)). Improving performance on attributes, however, requires careful decisions on which attributes deserve focused attention, and how resources should be allocated to numerous improvement efforts, especially when there are limited resources, which is often a challenging task of many companies. Instead of improving performance of all attributes, focusing on a core set of attributes that customer consider important or on attributes that are most impactful on customers' overall satisfaction, may be more prudent in maximizing the effectiveness of allocating limited resources. Equally important in this endeavor is to determine a target group of customer whom the improvement efforts should be focused on. Rather than making unilateral efforts on all customers, a carefully designed strategy that could be tailored for, and focused on those who may be most affected might be the key to maximizing the effectiveness of the improvement efforts.

\footnotetext{
${ }^{1}$ Note: This paper was presented at the 2015 International Business Conference in Maui.
} 
What, then, would be the most powerful attributes that could make the biggest impact on customers' overall satisfaction? How could the target customer group be identified, and the proper strategies, action plans and concerted efforts should be tailored to the critical needs of the target customer group? This study explores for possible answers for these questions, especially focusing on the second question, and proposes a method with which improvements efforts can be effectively prioritized and the effectiveness of resource allocation can be maximized.

\section{IDENTIFYING THE TRUE NATURE OF THE RELATIONSHIP BETWEEN SATISFACTION AND ATTRIBUTE PERFORMMANCE}

\subsection{Classification of Attributes}

Examining the non-linear nature of each attribute often provides a valuable insight with which the improvement efforts can be effectively tailored to the target customer group. Traditional approaches proposed by Anderson and Mittal (2000) and Mittal and Baldasare (1996) try to identify whether each attribute is satisfaction maintaining or satisfaction enhancing, and prioritize the improvement efforts on the basis of the nature of each attribute. These approaches assume that there is a nonlinear and asymmetric relationship between satisfaction and attribute performance, and recognize that the nature and extent of the asymmetry can be different for segmented customer groups. This indicates that the improvement efforts should be carefully managed and prioritized, and appropriate actions should be taken according to a noticeable of characteristic of an attribute. They suggest that failure to consider segment-specific differences may lead a firm to optimize performance on the wrong attribute for a given segment. When firms fail to consider segment-specific differences, they may often conclude that on average, an attribute is not important when, in fact, its importance may be artificially masked. Among the noteworthy contributions made by this study is to recognize the two different types of attributes that exhibit two distinctive forms of non-linear relationships.

Satisfaction-Maintaining Attributes (SMA) are core attributes that customer take for granted. Customers consider these attributes necessary and take for granted. The absence (or lack) of such attributes, in general, would result in customer dissatisfaction. As such, they are likely to exhibit negative asymmetry and diminishing returns in their impact on customer satisfaction, which results in a concave relationship. For these attributes, the impact of negative performance is typically greater than the equal amount of positive performance. Therefore, improvement efforts in the negative-performance domain have a greater impact on customer satisfaction than making similar efforts in the positive-performance domain.

Satisfaction-Enhancing Attributes (SEA) are the attributes that customers consider 'surprise', 'delightful', and 'fascinating'. Usually, customers do not anticipate huge performance improvement on such attributes because they are not usually related to what customers normally envision a product or service experience to entail. But the presence of such attributes would contribute significantly to improving customers' overall satisfaction. SatisfactionEnhancing Attributes typically exhibit a 'positive asymmetry' that results in a convex relationship, hence the improvement efforts should be focused more on the group of customers on the high-side of the curve (i.e., those who are satisfied or very satisfied). Improvement efforts toward the extreme of the upper end of the scale are more consequential than the efforts made in the middle or lower ranges (Anderson and Mittal (2000)). However, it is possible that an attribute could be neither satisfaction-enhancing nor satisfaction-maintaining. This may happen when a relationship between an attribute performance and overall satisfaction does not exhibit a pure form of convex or concave. Thus when determining attribute importance, attention should also be paid to segment-wise differences in the nature of the attribute. Although the traditional approaches have made significant contribution to the field of customer satisfaction research, they have also left a few unanswered questions with regard to how each attribute can be examined and identified as SMA or SEA. One of the drawbacks of the traditional approach also lies in the fact that the determination of the true nature (SMA or SEA) of non-linear relationships could be extremely difficult, and without an effective mechanism, the examination of all attributes could be prohibitively time-consuming. If the nature of the relationship is identified, focused improvement efforts can be carefully designed so that the impact of these efforts can be maximized. 


\subsection{The Background of the Proposed Approach}

In this section, we describe the conceptual background of the proposed method with which the nature (or any non-linear) of an attribute (or any attribute considered impactful) can be determined through a piecewise linear function without resorting to SMA or SEA. The method assumes that when the efforts are made to improve customers' satisfaction rating to a next higher rating, the percentage change of the affected customer groups can be utilized as a measure of determining the effectiveness of the improvement efforts. In general, assessing the impact of any improvement efforts on customer satisfaction may often require a longitudinal study and post-improvement analyses. In this study, the percentage change will be used as a measurable effect of the improvement efforts. Our proposed approach is also different from the traditional approach in that our approach would primarily focus on determining the group of targeted customers and their needs and concerns rather than on individual attribute and the nature of the relationship. A piecewise linear function will be also developed to observe the true nature of the nonlinear relationship.

Before we implement the proposed method, we assume that a list of critical attributes can be obtained and are available for the further analysis. While all attributes can be considered for the proposed approach, focusing on a smaller set of critical attributes that may have stronger influence on customers' overall satisfaction may maximize the effectiveness of the improvement efforts. Critical attributes can be identified, in general, on the basis of traditional measures such as average satisfaction ratings (attributes that receive the lowest satisfaction ratings), average importance ratings (attributes that receive the highest importance ratings), average gap ratings (attributes that show the largest gaps between importance ratings and satisfaction ratings) and/or some statistical methods such as Correlation Analysis (attributes that have the highest correlation with overall satisfaction) and Regression Analysis (attributes that are highly significant in affecting overall satisfaction). These methods are very popular and valuable in that they provide a good mechanism with which certain critical attributes can be easily determined.

After a set of critical attributes are determined, we move to the next step where customers' satisfaction with each critical attribute is examined and customers are segmented according to their satisfaction ratings with each critical attribute. At this step, we try to identify the percentages of customers who expressed their satisfaction with an attribute. For example, when a satisfaction survey uses a five-point scale ranging from 'very dissatisfied' (i.e., satisfaction rating of 1) to 'very satisfied' (i.e., satisfaction rating of 5), a percentage of customers who assigned each satisfaction rating is obtained. This information will be used to construct a cumulative piecewise function for each critical attribute where the horizontal axis represents each satisfaction rating and the vertical axis indicates a cumulative percentage of customers that includes those who assigned all lower satisfaction ratings. We believe that the target customer group can be determined by examining the differences of cumulative percentages between two breakpoints on a piecewise linear function. The improvement efforts on a critical attribute should be focused on a customer group who exhibits the highest difference.

\section{THE PROPOSED METHOD: THE METHODOLOGY}

\subsection{The Preliminaries}

Let $p_{i k}=\%$ of customers who assigned a rating of $k$ on attribute $i$, where $\mathrm{i}=1 \ldots \mathrm{m}, k=1 \ldots n$, and $\mathrm{m}$ represents the number of attributes (questionnaire items) on a survey and $n$ represents the number of ratings on a scale used in a survey.

Let $\mathrm{C}_{i k}=$ cumulative $\%$ of customers who assigned a rating of $k$ or lower on an attribute $i$. That is,

$$
\mathrm{C}_{i k}=\sum_{l=1}^{k} p_{i l} \text { and then, } \mathrm{C}_{i n}=\sum_{l=1}^{n} p_{i l}=100 \% \text { for each attribute } i
$$


Let $\Delta_{i k}=\mathrm{C}_{i k}-\mathrm{C}_{i k-1}$, where $i=1 \ldots m$ and $k=2 \ldots n . \Delta_{i k}$ represents a slope of each line segment that indicates the potential impact of improvement efforts. Then, by definition, when $\mathrm{k}=1, \Delta_{i 1}=p_{i 1}$, and $\Delta_{i \max }=\max$ $\left\{\Delta_{i 1} \ldots \Delta_{i n}\right\} . \Delta_{i \max }$ represents a line segment that has the steepest slope.

\subsection{The Proposed Method: A Step-by-Step Procedure}

The following step-by-step procedure describes the proposed method with which a target customer group can be identified, and where the improvement efforts should be focused on.

Step 1: We identify a list of critical attributes that require significant improvement efforts in improving customers' satisfaction level. Although the proposed method can be extended to any attributes including satisfactionmaintaining and satisfaction-enhancing attributes, our discussion will be limited to those considered 'critical.' As discussed in the previous section, the critical attributes can be determined based on any methods that the management considers appropriate for the situation. Average Importance Rating, Average Satisfaction Rating and Average Gap Rating are the most popular criteria that have been adopted by many companies. Regression Analysis and Correlation Analysis are also used in identifying significant attributes that may be strongly correlated with customers' overall satisfaction.

Step 2: For each critical attribute $i$, we determine the percentage (\%) of customers who assigned a satisfaction rating of k (i.e., $\left.p_{i k}\right)$.

Step 3: We identify the largest group of customers for whom the improvement efforts should be designed and implemented effectively. That is, we identify $p_{i \max }$, where $p_{i \max }=\operatorname{Max}\left\{p_{i 1} \ldots p_{i m}\right\}$, and ' $k$ ' where $p_{i k}=p_{i \max }$. The targeted group is the largest group of customers who assigned a satisfaction rating of ' $k$ '.

Step 4: Efforts are made to determine the critical needs and concerns of the targeted group of customers, and a strategy is developed to address those needs and concerns so that the effectiveness of the improvement efforts could be maximized.

\subsection{A Piecewise Linear Approximation for the Nonlinear Relationship}

As indicated in the previous section, Anderson and Mittal (2000) have pointed out the nonlinear nature of the relationship between overall satisfaction and attribute performance. In practice, however, it would be very difficult to identify pure forms of nonlinear functions (e.g., convex and concave functions) that would accurately represent the true relationships.

In this section, we introduce an approximation method that express the nonlinear relationship in terms of a piecewise linear function that properly represents the varying degrees of the different customer groups' satisfaction ratings. A piecewise linear function consists of several straight-line segments. In our example where there are five satisfaction ratings, there will be five line segments. The points where the slope of the piecewise linear function change are called the break points of the function. The cumulative percentage of the customer groups at each satisfaction rating will become the breakpoint. The slope, which is the difference between two consecutive break points, indicates the measurable impact of an improvement effort. For example, when an effort is made to improve the satisfaction level of the customer group from 'satisfied (rating of 4)' to the level of 'very satisfied (rating of 5)', the slope of the line segment in a piecewise linear function can be interpreted as a 'measurable improvement effect', and the target customer group will be those who assigned a rating of 4 . We recognize this slope as a potential impact of improvement efforts on customers' satisfaction with each critical attribute.

The piecewise linear function that utilizes the cumulative percentage of customer groups $\left(\mathrm{C}_{i k}\right)$ can provide a good estimate for the true relationship for each attribute. First, we identify the percentage of customers who 
assigned same satisfaction ratings on the attribute. And at each satisfaction rating, the cumulative percentage is obtained by finding the sum of individual percentage up to this specific satisfaction rating. The number of customer groups will be based on the scale used in the survey. When a customer's satisfaction with an attribute is measured on a 5-point scale (e.g., 1: very dissatisfied, 2: dissatisfied, 3: neutral, 4: satisfied, 5: very satisfied), there will be five such customer groups. Next, for each attribute, we calculate $\Delta_{i k}$ and $\Delta_{i \max }$. At this step, the percentage difference between two consecutive customer groups (breakpoints) is calculated, and the steepest slope (the largest difference) is identified. The information will be used in constructing a piecewise linear function for each attribute that may exhibit a certain relationship. $\Delta_{i \max }$ will enable us to determine the customer group on whom the improvement efforts should be focused. Finally, for each critical attribute, we construct a piecewise linear function that shows a relationship between each customer group (\%) and satisfaction ratings. The shape and slope at each line segment may help identify a target customer group (e.g., those who claimed 'Very Dissatisfied', 'Moderately Dissatisfied', 'Neutral', 'Moderately Satisfied' or 'Very Satisfied') that requires focused attention. The highest priority will be given to the customer group who has the largest slope with the highest percentage increase, which suggests that the greatest impact could be achieved and the effectiveness of utilizing the limited resources could be maximized.

\section{AN ILLUSTRATED EXAMPLE}

\subsection{Data Set}

Using a data set (sample size of 523) created from a user-satisfaction survey, we illustrate the proposed method. The survey measures students' satisfaction with a college laptop initiative. With the purpose of better understanding the true needs of students, who are the end-users of the laptop initiative, we carefully designed the questionnaire from the students' perspective by incorporating students' suggestions and concerns, rather than from the faculty or administrative staff perspectives. A 61-item survey questionnaire (55 importance/satisfaction items with 4 demographic questions and 2 more questions measuring (1) overall satisfaction and (2) whether the student would recommend the initiative to others based on their experience) was constructed to explore five themes in the areas of: (A) training and orientation support provided to adopters (13 questions), (B) end-user support (14 questions), (C) technology (6 questions), (D) economic issues (6 questions), and (E) enhancement of learning and use of laptops in classrooms (16 questions). Students were asked to rate their expectations and experiences with the laptop initiative with regard to "importance" and "satisfaction." The items were Likert-type statements on a fivepoint scale ranging from (1) Strongly Disagree to (5) Strongly Agree. At the end of the survey, a question regarding students' overall satisfaction was included on a five-point scale ranging from (1) Very Dissatisfied to (5) Very Satisfied. Students 'overall' satisfaction with the laptop initiative was utilized as a dependent variable in developing a regression model between overall satisfaction and satisfaction ratings.

\subsection{An Illustrated Example}

The proposed method is illustrated as follows. At step 1, the data obtained from the sample were carefully analyzed to determine the "critical attributes" on the basis of Average Gap Rating. The top three critical attributes were, in order of importance: (Attribute A) students' choice of what type of machine they want to purchase/lease, (Attribute B) adequate explanation of user fee, and (Attribute C) students' opportunity to upgrade the laptop (i.e., $i=\mathrm{A}, \mathrm{B}, \mathrm{C}$ ). At step 2 , for each attribute, customers are grouped in accordance with their satisfaction ratings on the attribute, and for each customer group, a percentage of each customer group $\left(p_{i k}\right)$ was obtained. Five such customer groups are included in this example because of a five-point scale used in the survey (i.e., $k=1,2,3,4,5)$. Table 1 shows the values of $p_{i k}$ and $\mathrm{C}_{i k}$. At Step 3, we identify $p_{i \max }=\operatorname{Max}\left\{p_{i 1} \ldots p_{i m}\right\}$. Take Attribute A for example, $p_{A \max }=\operatorname{Max}\left\{p_{A 1} \ldots p_{A m}\right\}=$ $\operatorname{Max}\{34.02,23.68,22.18,10.53,9.59\}=34.02=p_{A 1}$. The maximum has occurred at the first group of customer. Hence, ' $\mathrm{k}$ ' $=1$. The targeted group of customers is those who are 'very dissatisfied' with Attribute A. At the final step, the improvement efforts should be focused on 'Group 1' to determine why they are 'very dissatisfied' and what their concerns, needs and wants are, and how the university can better address these issues with limited resources. 
In order to observe the nature of the non-linear relationship of Attribute $\mathrm{A}$, we utilize $p_{A k}$ and $\mathrm{C}_{A k}$ provided in Table 1. We note that $34.02 \%$ of students assigned a rating of 1 (Very Dissatisfied), which indicates that $\Delta_{A 1}=p_{A 1}=\Delta_{A \max }=34.02$. We also note that the steepest slope in the piecewise linear function occurs at customer group 1. Judging from this observation, we could conclude that the improvement efforts for Attribute A should be focused on customers who are very dissatisfied. Overall $57.7 \%$ of customers are very dissatisfied or dissatisfied with Attribute A. Determining the critical needs of these customer groups and addressing their concerns would make the improvement efforts for more effective and impactful than simply focusing on all customer groups or satisfied customers. A piecewise linear function for Attribute A is shown in Figure 1. Similar conclusion can be drawn for Attribute B. Table 1 shows that $\Delta_{B 1}=p_{B 1}=\Delta_{B \max }=28.02$, which indicates that improvement efforts should be focused on groups of customers who are very dissatisfied or dissatisfied with Attribute B rather than on those who are satisfied.

Unlike Attributes A and B, Attribute $\mathrm{C}$ presents a different perspective. Table 1 indicates that $p_{C \max }=$ Max $\left\{p_{C 1} \ldots p_{C m}\right\}=\operatorname{Max}\{23.0,23.75,34.75,13.13,5.37\}=34.75=p_{C 3}$. And $\Delta_{C \max }=\Delta_{C 3}=34.75$. A satisfaction rating of 3 (i.e., ' $\mathrm{k}$ ' $=3$ ) represents a group of customers who are neutral to Attribute $\mathrm{C}$ with regard to their satisfaction level. Identifying the concerns and critical needs of this group and moving them to a higher level of satisfaction will present a daunting task for the management.

In general, the overall shape of the piecewise function and corresponding slopes at line segments provide intuition to whom improvements efforts should be focused on and how such efforts should be customized.

Table 1. Individual and Cumulative \% of Customer Groups for Critical Attributes

\begin{tabular}{|c|c|c|c|c|c|c|}
\hline \multirow{3}{*}{$\begin{array}{c}\text { Satisfaction } \\
\text { Rating }\end{array}$} & \multicolumn{6}{|c|}{ Attributes } \\
\hline & \multicolumn{2}{|c|}{$\mathbf{A}$} & \multicolumn{2}{|c|}{ B } & \multicolumn{2}{|c|}{$\mathrm{C}$} \\
\hline & $\begin{array}{c}\text { \% of } \\
\text { Customers }\left(p_{i k}\right)\end{array}$ & $\begin{array}{c}\text { Cum. \% } \\
\left(\mathrm{C}_{i k}\right)\end{array}$ & $\begin{array}{c}\text { \% of } \\
\text { Customers }\left(p_{i k}\right)\end{array}$ & $\begin{array}{c}\text { Cum. \% } \\
\left(\mathrm{C}_{i k}\right)\end{array}$ & $\begin{array}{c}\text { \% of } \\
\text { Customers }\left(p_{i k}\right)\end{array}$ & $\begin{array}{c}\text { Cum.\% } \\
\left(\mathrm{C}_{i k}\right)\end{array}$ \\
\hline 1 & 34.02 & 34.02 & 28.02 & 28.02 & 23 & 23 \\
\hline 2 & 23.68 & 57.7 & 24.18 & 52.2 & 23.75 & 46.75 \\
\hline 3 & 22.18 & 79.88 & 22.84 & 75.04 & 34.75 & 81.5 \\
\hline 4 & 10.53 & 90.41 & 16.31 & 91.35 & 13.13 & 94.63 \\
\hline 5 & 9.59 & 100 & 8.65 & 100 & 5.37 & 100 \\
\hline
\end{tabular}

Note: Attribute A represents 'Students want to have the choice of what type of machine they want to purchase/lease'. Attribute B represents 'Adequate explanation of user fee'. Attribute C represents 'Students have the opportunity to upgrade the laptop'.

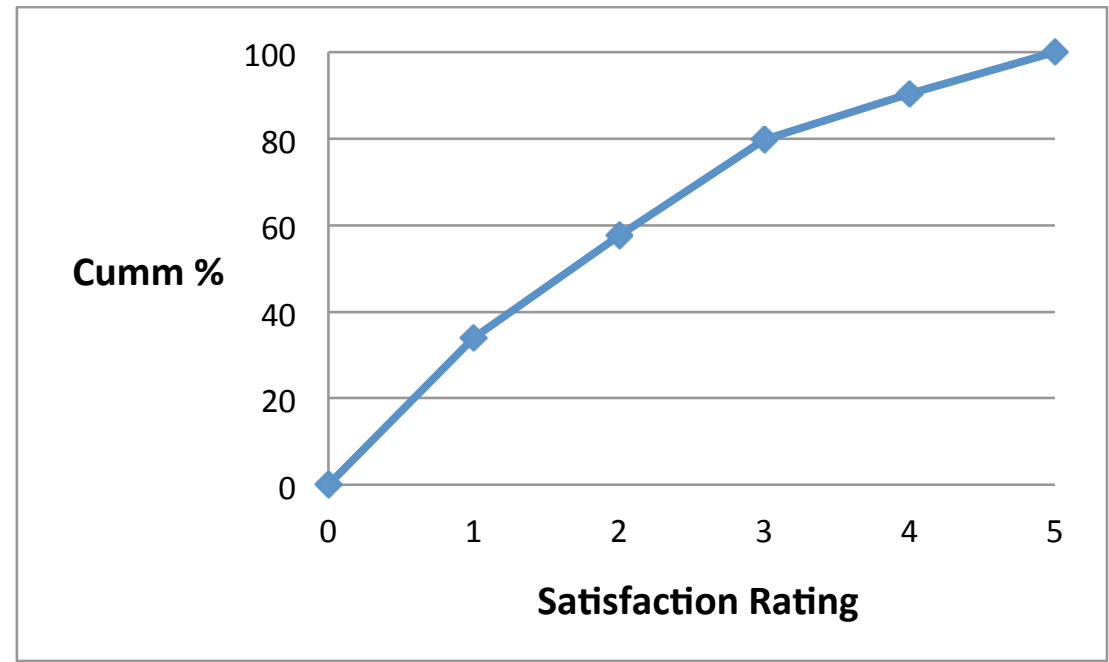

Figure 1. The Piecewise Linear Function: Attribute A 


\subsection{Discussion}

As we discussed in the previous sections, the overall shape of the piecewise linear functions can be used in understanding and estimating the nature of non-linear relationship of each attribute. The concave nature of the piecewise linear functions in Figures 1 and 2 indicates that Attributes A and B are Satisfaction-Maintaining attributes. As indicated in Table 1, more customers are very dissatisfied with these attributes, which suggests that improvement efforts should focus more on these groups in order to have a greater impact on customer satisfaction than making similar efforts on satisfied customers. Especially because these attributes are considered necessary and must-be-in attributes, and can result in customer dissatisfaction, improvement efforts should center on dealing with the critical needs and concerns of the very dissatisfied group of customers. The piecewise linear function in Figure 3 suggests that Attribute $\mathrm{C}$ is neither concave (Satisfaction-Maintaining) nor convex (Satisfaction-Enhancing). For this attribute, far greater impact can be achieved when concerted efforts are mounted on the group of neutral customers rather than on the satisfied customers.

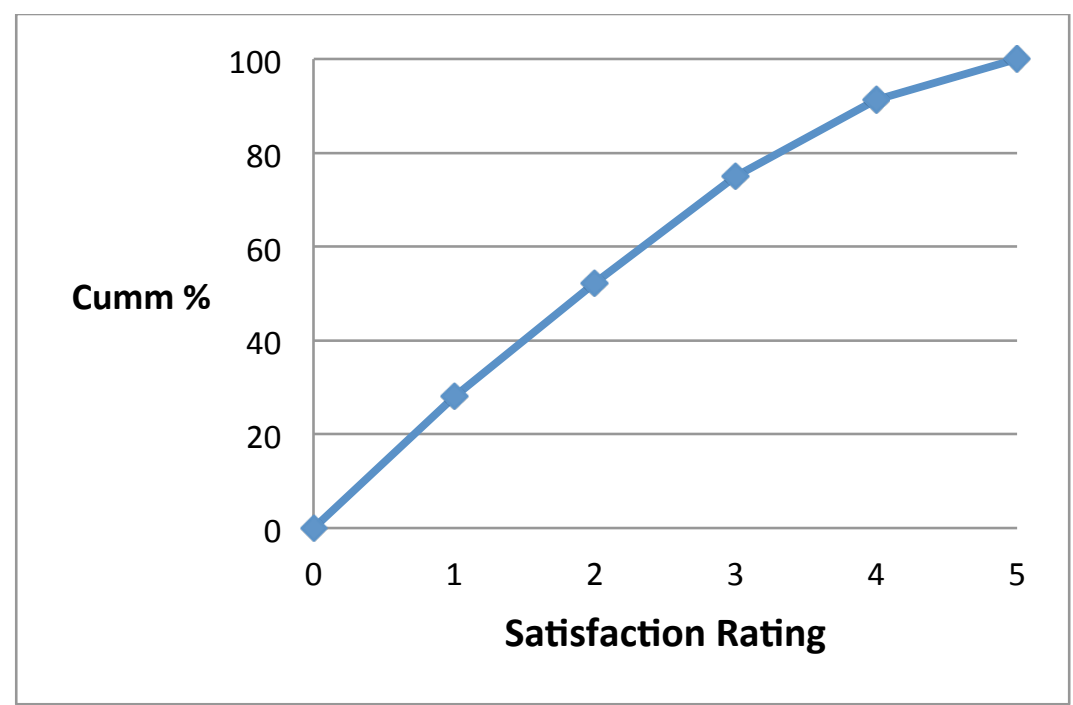

Figure 2: The Piecewise Linear Function: Attribute B

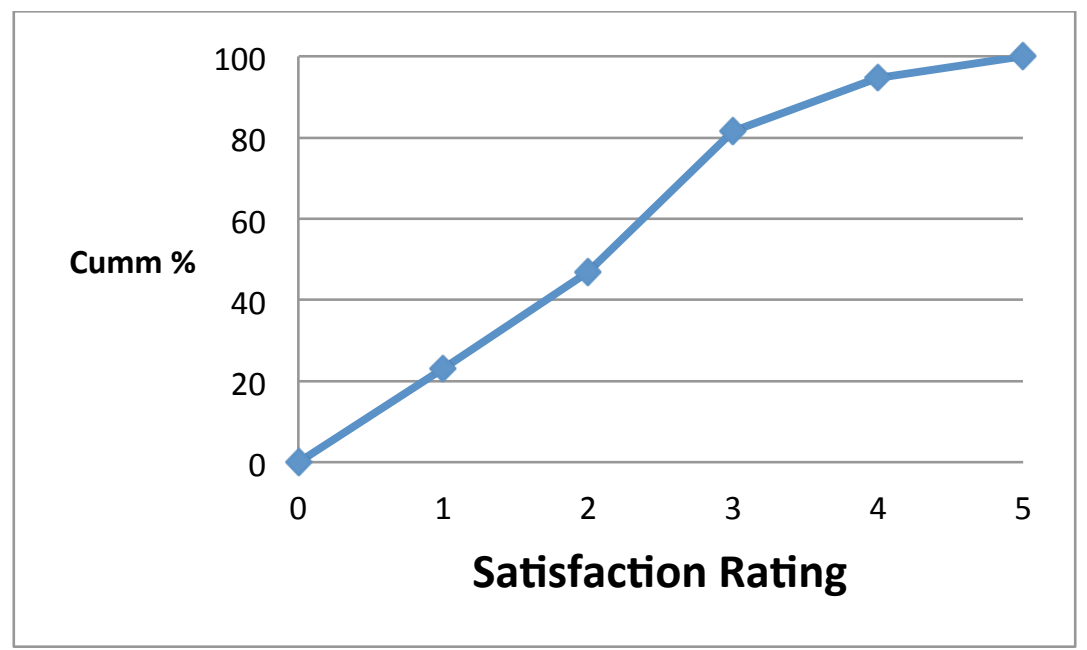

Figure 3: The Piecewise Linear Function: Attribute C 


\section{SUMMARY AND CONCLUSIONS}

Recognizing that customer satisfaction with a product or service could be a key determinant of the profitability, companies make strenuous efforts to improve customer satisfaction. As is often the case, many companies struggle with developing a proper strategy in allocating limited resources to such improvement efforts. Improvement efforts are often misdirected and ill-focused, and failed to generate a corresponding improvement in customer satisfaction. This phenomenon happens when there is a mismatch between the needs of the targeted customers and the direction of the improvement efforts. This paper proposed a method with which the improvement efforts could be better focused, and could address the critical needs of the targeted customers more effectively. The proposed approach tries to determine a target customer group to whom the improvement efforts should be concentrated. Once a targeted group of customers is identified, the critical needs of this group can be determined, and a proper strategy and an appropriate action plan can be developed and implemented. This paper also proposed an approximation method with which a non-linear nature of an attribute can be estimated with a construction of piecewise linear function. Even though determining the true nature of any non-linear relationship (e.g., SatisfactionMaintaining or Satisfaction-Enhancing) is often a very daunting task, the proposed method can be utilized in understanding and estimating the non-linear relationship.

\section{AUTHOR INFORMATION}

Dr. Dooyoung Shin earned his Ph.D. at the University of Iowa, Iowa City in 1987. He is currently a professor of operations management at Minnesota State University, Mankato. His teaching and research interests include operations management, quality management and management science. E-mail: Dooyoung.shin@mnsu.edu (corresponding author)

Dr. Jon Kalinowski earned his Ph.D. at the University of Iowa, Iowa City in 1987. He is currently a professor of strategic management at Minnesota State University, Mankato. His teaching and research interests include strategic management, leadership and motivation theory, quality-related initiatives and executive succession. Email: jon.kalinowski@mnsu.edu

Dr. Sung Kim earned his Ph.D. at the University of Nebraska, Lincoln in 1995. He is currently an associate professor of management information system at Minnesota State University, Mankato. His teaching and research interests include MIS, decision support systems and end-user satisfaction. E-mail: sung.kim@mnsu.edu

Dr. In-Jae Kim earned his Ph.D. at the University of Wyoming. He spent a year at University of Victoria, BC, Canada as a postdoctoral fellow before he joined the Department of Mathematics and Statistics at Minnesota State University, Mankato. He is currently an associate professor of mathematics and a research fellow at the MN Modeling and Simulation Center at Minnesota State University, Mankato. He also serves as the Director of Center for Excellence in Scholarship and Research at Minnesota State University, Mankato. His teaching and research interests include combinatorial matrix theory, graph theory, and complex network theory.

E-mail: in-jae.kim@mnsu.edu

\section{REFERENCES}

Anderson, Eugene W. and Mittal, Vikas (2000). Strengthening the satisfaction-profit chain. Journal of Service Research, 3(2), 107-120.

Bitner, M.J. and Hubert, A.R. (1994). Encounter satisfaction versus overall satisfaction versus quality. In Rust, R.T. and Oliver R.L. (eds), Service quality: new directions in theory and practice, Sage, Thousand

Oaks, CA. 72-94.

Borden, Victor M.H. (1995). Segmenting student markets with a student satisfaction and priorities survey," Research in Higher Education, 36 (1), 73-88.

Brown, Stephen W. and Teresa A. Swartz (April 1989). A gap analysis of professional service quality. Journal of Marketing, Vol. 53, 92-98.

Hanson, Randy. (October 1992). Determining attribute importance. Quirk's Marketing Research Review, Vol. 6, 16-18. 
Hartman, David E. and Sandra L. Schmidt. (1995). Understanding student/alumni satisfaction from a consumer's perspective: the effects of institutional performance and program outcomes. Research in Higher Education, 36 (2), 197-217.

Jones, A. M. and Suh, J. (2000). Transaction-specific satisfaction and overall satisfaction: an empirical analysis. Journal of Services Marketing, 14 (2), 147-159.

McColl-Kennedy, J. and Schneider, U. (2000). Measuring customer satisfaction: why, what and how. Total Quality Management, 11 (7), 883-896.

Mittal, Vikas and Patrick M. Baldasare. (1996). Eliminate the negative. Journal of Health Care Marketing, 16 (3), $24-$

Mittal, V., Ross W. T. and P. Baldasare. (1998). The asymmetric impact of negative and positive attribute-level performance on overall satisfaction and repurchase intentions. (1998). Journal of Marketing, Vol. 62, 33-47.

Shin, D., Kalinowski, J., \& Kim, S. (2004). Identifying key drivers of students' overall satisfaction. Review of Business Research, 2 (1), 150-161.

Shin, D., Kalinowski, J., \& Kim, S. (2007). Measurement of end-users satisfaction in a college laptop initiative. Review of Business Research, 7 (4), 146-156.

Shin, D., Kalinowski, J., \& Kim, S., "Overall Satisfaction Measurement: An Improved Multi-Attributes Method," Journal of International Business Research, Vol. 10 (5), 2010, 196-203.

Vavra, G. Terry. (1997). Improving Your Measurement of Customer Satisfaction, Quality Press, Milwaukee, WI.

Wittink, Dick R. and Leonard R. Bayer. (1994). The measurement imperative. Marketing Research, 6 (4), $14-23$.

Yi, Youjae. (1990). A critical review of consumer satisfaction. in Review of Marketing, Valarie A. Zeithaml, ed. Chicago: American Marketing Association, 68-123.

Zeithaml, Valarie A. (2000). Service quality, profitability, and the economic worth of customers: what we know and what we need to learn. Journal of the Academy of Marketing Science, 28(1), 67-85. 
The Journal of Applied Business Research-May/June 2015

Volume 31, Number 3

NOTES 